\title{
Generalization of serial learning in the pigeon
}

\author{
R. O. STRAUB \\ University of Michigan, Dearborn, Michigan 48128 \\ and \\ H. S. TERRACE \\ Columbia University, New York, New York 10027
}

\begin{abstract}
Sequence learning in pigeons was studied in a simultaneous chaining paradigm: all stimuli and the opportunity to respond to each stimulus were available simultaneously. In contrast to the traditional successive chaining paradigm, a simultaneous chaining paradigm provides no differential feedback following each response (except the last). Subjects were first trained to perform on sequences of two (AB), then three (ABC), and then four colors (ABCD). Performance greatly exceeded that predicted by models of random choice. Generalization to novel arrays of three and four colors was complete. After training with a four-color sequence, the subjects were tested with subsequences consisting of all possible combinations of two and three of the four training colors (e.g., BD, AD, BC, ACD, BCD, etc.). The successful completion of these subsequences showed that the organization of the original sequence did not entail overt pecks to successive elements of that sequence. That subjects can respond accurately on nonadjacent subsets is not readily explained by a chaining theory, or by any theory that assumes that responding to element $n$ provides a cue for responding to element $n+1$.
\end{abstract}

Serially organized action is the norm in animal and human behavior. Most animal research, however, has focused on single responses. Even when sequences have been studied (e.g., in mazes or in chained schedules), behavior organization has been explained as chains of individual responses. While learning theorists (Guthrie, 1952; Hull, 1943; Skinner, 1938) have invoked different forms of this explanation, they have each argued that a particular sequence of behavior can be completely reduced to a sequence of S-R units: the occurrence of $R_{n}$ produces $S_{n+1}$, which elicits (or occasions) $R_{n+1}$, and so on. This view suggests that an organism that learns a sequence of responses has simply learned to respond appropriately to a series of successively presented stimuli, and nothing more. For example, a rat that learns to run through a maze need not have any knowledge of the plan of the maze. At the first choice point, it makes a response appropriate to $S_{1}$; that response, $R_{1}$, is followed by the appearance of $S_{2}$, and so on.

Chaining theory has been challenged on a number of grounds as an explanation of integrated sequences of human behavior (Broadbent, 1961; Chomsky, 1957, 1959; Lashley, 1951; Lashley \& Ball, 1929;

This paper is based, in part, on a doctoral dissertation submitted to Columbia University by $R$. $O$. Straub. The research was supported in part by grants from NSF (BNS72-U128A123176), NIMH (HD-00930), and the Harry Frank Guggenheim Foundation. Requests for reprints should be sent to $\mathrm{H}$. S. Terrace, Columbia University, 418 Schermerhorn Hall, New York, New York 10027.
Miller, Galanter, \& Pribram, 1960). Lashley noted that, in many human behavioral sequences, selfgenerated proprioceptive stimuli provide the only source of step-by-step feedback, for example, in typing or in playing a musical instrument. In these instances, the intervals between successive responses are too brief to allow the proprioceptive consequences of $R_{n}$ to function as a discriminative stimulus for $R_{n+1}$. Another of Lashley's arguments, subsequently elaborated by Chomsky, revealed the weakness of a chaining model in explaining sentences, such as this one, in which relationships exist between nonadjacent words.

Until recently, only the realm of human performance provided clear examples of sequential behavior that were difficult to explain by reference to chaining theory. During the past few years, however, a number of examples of animal performance have appeared that also seem refractory to explanations based on chaining theory.

In a series of experiments employing a radial maze, Olton showed that rats were able to remember which arms of the maze they had visited, without any apparent mediation by exteroceptive or proprioceptive cues (Olton, 1978, 1979; Olton \& Samuelson, 1976). Each arm of the maze ( 8 in some of the experiments, 17 in others) was baited with food. With relatively little training, the rat learned to obtain food at the end of each alley without returning to alleys that it had emptied earlier. Olton ruled out olfactory cues as an explanation of such performance. He also showed 
that the sequence of alley entries varied randomly from trial to trial and thereby demonstrated that the rat was not using a stereotyped chain in entering successive arms of the maze. Olton concluded that the rat relied on a spatial representation of the maze in deciding which arm to enter. It is unclear how a spatial representation could be derived from a chaining theory that requires a distinctive stimulus to precede each response.

Another study employed a simultaneous chaining paradigm, a modification of the traditional chaining procedure, to demonstrate that pigeons could learn a "list" of colored lights (Straub, Seidenberg, Bever, \& Terrace, 1979; Terrace, Straub, Bever, \& Seidenberg, 1977). In a conventional successive chaining paradigm, each correct response produces feedback that results in the presentation of the next discriminative stimulus (and, when necessary, the appropriate manipulandum). A simultaneous chaining paradigm provides no step-by-step feedback following each response.

In the Terrace et al. study (described in detail by Straub et al.), pigeons were trained to peck 15 different arrays of four colors $(A, B, C, D)$ in the sequence $A \rightarrow B \rightarrow C \rightarrow D$, regardless of how those colors were spatially positioned on four response keys. Four new arrays were presented following training on the 15 original $A B C D$ arrays. Responding to these arrays was significantly better than chance. This finding indicates that the high level of accuracy observed on the 15 training arrays could not have resulted solely from memorization of the different configurations that defined the various arrays.

In interpreting the results of this study, it is important to note that food reinforcement was provided only after the final response (to color $D$ ). No programmed consequences followed correct responses to colors A, B, or C. Forward errors (e.g., a peck to $D$, after pecks to $A$ and $B$ ) and backward errors (e.g., a peck to $A$ after a peck to $A$ and $B$ ) did result in the immediate termination of the trial. Chaining theory cannot, however, explain correct sequences of responses solely on the basis of feedback that follows errors.

In the absence of control by successively presented exteroceptive stimuli, it is necessary to consider what internal representations the pigeon uses in performing the four-element sequence as trained by Straub et al. As in the case of studies of representation by humans, specifying the nature of a representation proves much more difficult than simply demonstrating a need to postulate a representation (Fodor, Bever, \& Garrett, 1974; Riley, 1976; Shepard, 1975). The present study seeks to provide a basis for characterizing the representation(s) used by a pigeon in the performance of a simultaneous chain.

\section{EXPERIMENT 1}

The purpose of Experiment 1 was to clarify two features of the data described in our earlier study (Straub et al., 1979). One problem resulted from the numerous changes in procedure that were made while training on the simultaneous chain. These changes prevented us from presenting an orderly picture of acquisition. In studying the acquisition of a simultaneous chain, we also sought to obtain systematic data concerning various parameters of performance at accuracy levels of $25 \%, 50 \%$, and $70 \%$ (relative frequencies and latencies of correct responses, forward and backward errors, and repeat responses). A second problem with the Straub et al. data was the generalization decrement observed when novel arrays were introduced. While performance to novel arrays was reliably better than would be expected by chance, the fact that any decrement occurred suggested that some degree of learning of the 15 training arrays had taken place.

Two changes of procedure were introduced, one to minimize the possibility of learning to respond to specific physical configurations of colors and the other to obtain a clearer picture of the generalization of serial learning. Eighty (rather than 15 ) training arrays were used and 20 (rather than 4) novel arrays were used during the generalization tests that followed three-element $(A, B, C)$ and four-element $(A, B, C, D)$ training.

\section{Method}

Subjects

The subjects were eight White Carneaux pigeons, maintained at $80 \% \pm 3 \%$ of their free-feeding weights. All birds were at least 1 year old. Prior to the beginning of the experiment, each subject was trained for several weeks on a variety of single-key autoshaping paradigms.

\section{Apparatus}

Training and testing were conducted in a six-key operant chamber. The front panel of the chamber consisted of two rows of three translucent response keys, $2.54 \mathrm{~cm}$ in diameter and $6.67 \mathrm{~cm}$ apart, center-to-center. The top row was centered $26.35 \mathrm{~cm}$ above the mesh floor of the chamber and $4.13 \mathrm{~cm}$ above the bottom row of keys. Each key required a force of approximately $15 \mathrm{~N}$ for closure of the microswitch. Beneath the keys and $10.16 \mathrm{~cm}$ above the floor were centered three food hoppers; their openings were each $5.08 \mathrm{~cm}$ square and $7.62 \mathrm{~cm}$ apart. In this study, only the center hopper was operable. The test panel was housed in a ventilated Industrial Acoustics Company sound-attenuated chamber (Model 102580) located in a sound-attenuated room. Continuous white noise served to mask extraneous sounds. A PDP/8-e computer located in a separate room controlled the experiment and recorded the data.

Stimuli were rear-projected onto the keys by a Kodak Carousel projector. The slides were prepared by photographing highly saturated construction papers (blue, red, green, and yellow), under BBA bulb illumination (color temperature $=3400 \mathrm{~K}$ ), with Kodak KPA Kodachrome film. The colors were arranged in a $2 \times 3$ matrix, 
each cell corresponding to one of the six response keys on the test panel. Each slide contained 1,2, 3, or all 4 of the colors in varying positions within the matrix. The colors appeared equally luminous to the experimenters' eyes when projected onto the response keys.

\section{Procedure (Group 1)}

Following magazine training, four subjects were autoshaped to each of the four colors: A (blue), B (red), C (green), and D (yellow). During autoshaping, each of the four colors was presented individually on each of the six keys in an irregular sequence. Each trial consisted of a 5-sec presentation of one color followed by reinforcement. The chamber was illuminated by a $15-\mathrm{W}$ houselight except during reinforcement. The intertrial interval (ITI) was variable (mean $I T I=15 \mathrm{sec}$ ). Except where noted, all sessions throughout the experiment were terminated after 80 trials. Autoshaping training was continued daily until each bird responded on $70 \%$ of the trial.

$\mathbf{A} \rightarrow \mathbf{B}$ successive training. Once the birds were responding reliably to all colors, colors A and B were presented successively, on different keys, during each trial. Each trial began with the presentation of $\mathbf{A}$ on one of the six keys. A single peck to $\mathbf{A}$ resulted in the termination of color $\mathbf{A}$ and the presentation of color $\mathbf{B}$, alone, $.5 \mathrm{sec}$ later. A peck to $B$ (within $15 \mathrm{sec}$ of its presentation) terminated the trial and activated the food hopper for $4 \mathrm{sec}$. Each bird received one 40-trial session of $A \rightarrow B$ successive training.

$A \rightarrow B, A \rightarrow B \rightarrow C$, and $A \rightarrow B \rightarrow C \rightarrow D$ simultaneous training. Following successive $(A \rightarrow B)$ training, colors $A$ and $B$ were presented simultaneously during each trial. The simultaneous $A \rightarrow B$ arrays consisted of each of the 30 possible combinations of the two colors on the six keys. A trial consisted of a $15-\mathrm{sec}$ illumination of two of the six keys with colors A and B. Reinforcement was contingent upon pecking $A$ and $B$ in the sequence $A \rightarrow B$, regardless of the keys on which $A$ and $B$ were presented. During this and all subsequent phases of training, repetitive pecks were not considered errors. For example, the sequence of pecks $A \rightarrow A \rightarrow A \rightarrow A \rightarrow B$ was considered correct. A correct sequence of responses to $A$ and $B$ terminated the array and produced reinforcement. Each response, correct or not, was followed by a feedback click that was generated by a relay mounted on the back of the panel.

During this and all subsequent phases of training, a trial was terminated following an error (in this case, an initial peck to B) or by the failure to complete the required sequence within $15 \mathrm{sec}$ of the onset of the trial. Errors also initiated a 4-sec time-out (TO), during which the chamber was darkened. For each subject, a particular stage of simultaneous training $(A \rightarrow B, A \rightarrow B \rightarrow C$, or $A \rightarrow B \rightarrow$ $C \rightarrow D$ ) continued until the following criterion was met: correct completion of $70 \%$ of the trials on two consecutive sessions. During $A \rightarrow B \rightarrow C$ training, a fixed sequence of 80 different arrays was presented during each session. These were chosen randomly from a set of 120 possible configurations of $A, B$, and $C$ on the six keys. During $A \rightarrow B \rightarrow C \rightarrow D$ training, each session consisted of a fixed sequence of 80 different arrays. These were selected randomly from the 360 possible arrays that can be generated by locating four colors on six response keys.

$A \rightarrow B \rightarrow C$ and $A \rightarrow B \rightarrow C \rightarrow D$ Generalization Tests. In the session after the $70 \%$ criterion was satisfied during $A \rightarrow B \rightarrow C$ training, generalization to novel arrays was tested by replacing $25 \%$ of the training arrays with novel arrays. The test session was identical to an $A \rightarrow B \rightarrow C$ training session, with the exception that every fourth training array $(N=20)$ was replaced with an array that was selected randomly from a set of $\mathbf{4 0}$ arrays not used during regular training. An $A \rightarrow B \rightarrow C \rightarrow D$ generalization test was given during the first session after the $50 \%$ and the $70 \%$ criteria were satisfied. During each test session, every fourth training array was replaced with an array $(N=20)$ selected at random from the 280 arrays of four colors that had not been employed during training. Different sets of novel arrays were used for each test session. During each generalization test, contingencies for correct responses and errors were identical to those in effect during the training sessions.

\section{Procedure (Group 2)}

A second group of subjects received training that was modeled after studies of human memory (e.g., Primoff, 1938) in which serial learning of a list of elements (e,g., A,B,C,D) appeared to be facilitated by prior paired-associate learning of adjacent elements (e.g., AB, BC, and CD). Four pigeons were trained on the color pairs $\mathrm{AB}, \mathrm{BC}$, and $\mathrm{CD}$ following autoshaping to $\mathrm{A}, \mathrm{B}, \mathrm{C}$, and $D$ (and one $A \rightarrow B$ successive session). Each color-pair session consisted of 80 trials in which 27,27 , and 26 trials of $A \rightarrow B, B \rightarrow C$, and $C \rightarrow D$ arrays, respectively, were presented. Reinforcement was contingent upon the following sequences of responses: $A \rightarrow B$, $\mathrm{B} \rightarrow \mathrm{C}$, and $\mathrm{C} \rightarrow \mathrm{D}$. Repetitive responses to the first member of each color pair were allowed. Errors were defined as pecking B, C, or $D$ prior to $A, B$, and $C$, respectively, and resulted in a 4-sec TO. Immediately following this phase of training, each bird went through the same training sequence given to the subjects of Group 1, beginning with successive $A \rightarrow B$ training. Table 1 summarizes the training histories of Groups 1 and 2.

\section{Results}

Seven of the eight subjects performed accurately on two-, three-, and four-color sequences at levels that greatly exceeded those predicted by a model of random choice responding. However, accuracy of responding decreased precipitously following the transitions from $A \rightarrow B$ to $A \rightarrow B \rightarrow C$ training and from $A \rightarrow B \rightarrow C$ to $A \rightarrow B \rightarrow C \rightarrow D$ training. Pretraining on $A B, B C$, and $C D$ pairs had no effect on the acqui-

Table 1

Training Histories of Subjects

\begin{tabular}{|c|c|c|c|c|c|c|c|c|}
\hline & \multicolumn{7}{|c|}{ Bird Number } & \multirow[t]{2}{*}{. } \\
\hline & \multicolumn{4}{|c|}{ Group 1} & \multicolumn{3}{|c|}{ Group 2} & \\
\hline & 262 & 263 & 259 & 258 & 298 & 299 & 203 & 296 \\
\hline A, B, C, D Autoshaping & $\mathbf{x}$ & $\mathbf{x}$ & $\mathbf{x}$ & $\mathbf{x}$ & $\mathbf{x}$ & $\mathbf{x}$ & $\mathbf{x}$ & $\mathbf{x}$ \\
\hline$A \rightarrow B$ Successive & $\mathbf{x}$ & $\mathbf{x}$ & $\mathbf{x}$ & $\mathbf{x}$ & $\mathbf{x}$ & $\mathbf{X}$ & $\mathbf{x}$ & $\mathbf{x}$ \\
\hline$A \rightarrow B, B \rightarrow C, C \rightarrow D$ Pretraining & & & & & $\mathbf{x}$ & $\mathbf{x}$ & $\mathbf{x}$ & $\mathbf{x}$ \\
\hline$A \rightarrow B$ Simultaneous & $\mathbf{x}$ & $\mathbf{x}$ & $\mathbf{x}$ & $\mathbf{x}$ & $\mathbf{x}$ & $\mathbf{x}$ & $\mathbf{x}$ & $\mathbf{x}$ \\
\hline$A \rightarrow B \rightarrow C$ Simultaneous & $\mathbf{x}$ & $\mathbf{x}$ & $\mathbf{x}$ & & $\mathbf{x}$ & $\mathbf{x}$ & $\mathbf{x}$ & $\mathbf{x}$ \\
\hline$A \rightarrow B \rightarrow C 70 \%$ Generalization Test & $\mathbf{x}$ & $\mathbf{x}$ & $\mathbf{x}$ & & $\mathbf{x}$ & $\mathbf{x}$ & $\mathbf{x}$ & $\mathbf{x}$ \\
\hline$A \rightarrow B \rightarrow C \rightarrow D$ Simultaneous & $\mathbf{x}$ & $\mathbf{x}$ & $\mathbf{x}$ & & $\mathbf{x}$ & $\mathbf{x}$ & $\mathbf{x}$ & $\mathbf{x}$ \\
\hline $\mathrm{A} \rightarrow \mathrm{B} \rightarrow \mathrm{C} \rightarrow \mathrm{D} 50 \%$ Generalization Test & $\mathbf{x}$ & $\mathbf{x}$ & $\mathbf{x}$ & & $\mathbf{x}$ & $\mathbf{x}$ & $\mathbf{x}$ & $\mathbf{x}$ \\
\hline$A \rightarrow B \rightarrow C \rightarrow D 70 \%$ Generalization Test & $\mathbf{x}$ & $\mathbf{x}$ & $\mathbf{x}$ & & $\mathbf{x}$ & $\mathbf{x}$ & & \\
\hline
\end{tabular}




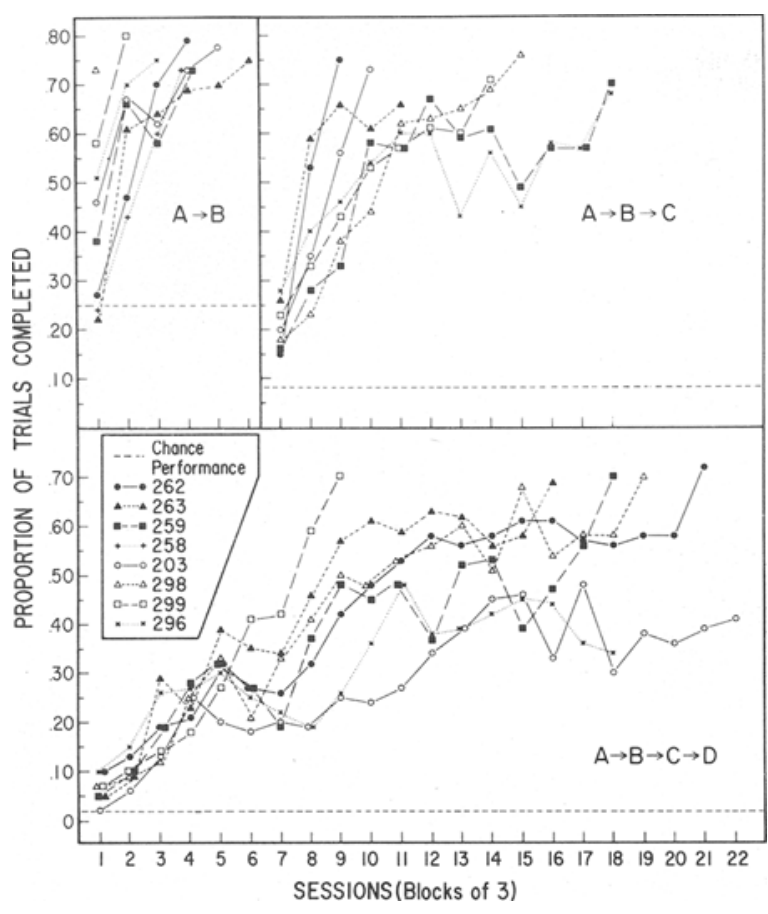

Figure 1. Proportion of trials, for each subject, completed correctly: $A \rightarrow B$ (upper left panel), $A \rightarrow B \rightarrow C$ (upper right panel), and $A \rightarrow B \rightarrow C \rightarrow D$ (bottom panel).

sition of $A \rightarrow B, A \rightarrow B \rightarrow C$, or $A \rightarrow B \rightarrow C \rightarrow D$ sequences. Throughout training, the relative frequency of forward errors greatly exceeded that of backward errors. Accuracy of performance on a particular element was not influenced by the occurrence of one or more repeat pecks to a prior element. All subjects showed complete generalization from training arrays to novel arrays of three and four colors.

\section{Acquisition}

$\mathbf{A} \rightarrow \mathbf{B}$ training. All subjects met a criterion of correct completion of at least $70 \%$ of $A \rightarrow B$ trials during two consecutive sessions (cf. Figure 1, upper lefthand panel). Accuracy at chance level can be calculated with and without allowance for repeat pecks to a given color. Since all of our subjects made repetitive responses to all but the last element of the sequence, the chance level of accuracy to which we compared our results allowed for repeats. Chance level of performance (.25) during $\mathrm{A} \rightarrow \mathrm{B}$ training was calculated as follows: $\mathrm{p}$ (correct first choice) $=.5$; $\mathrm{p}$ (correct second choice) $=.5$; $\mathrm{p}$ (correct $\mathrm{A} \rightarrow \mathrm{B}$ sequence) $=.5 \times .5=.25$. The difference between the performances of our subjects at the end of $A \rightarrow B$ training and that expected by chance was highly significant $[t(7)=15.93, p<.0005]$.

No difference was observed in mean trials required to achieve criterion by Group 1 (11 sessions) and Group 2 (6.50 sessions) $[\mathrm{t}(6)=1.52, \mathrm{p}>.10]$, al- though three of the four subjects in Group 2 reached criterion earlier than any subject in Group $1 .^{1}$

$\mathbf{A} \rightarrow \mathbf{B} \rightarrow \mathbf{C}$ training. Seven of our eight subjects met the criterion of completing at least $70 \%$ of $A \rightarrow B \rightarrow C$ trials during two consecutive sessions (cf. Figure 1, upper right). One subject of Group 1 (No. 258) was dropped from the study after the first five $A \rightarrow B \rightarrow C$ sessions for failure to respond.

Chance level of accuracy for completing correctly an $\mathrm{A} \rightarrow \mathrm{B} \rightarrow \mathrm{C}$ sequence $=.33 \times .50 \times .50=.085$. (Following the first response there exists only one way of making an error. This is because successive responses to the same stimulus were not counted as errors.) At the end of $A \rightarrow B \rightarrow C$ training, the performance of our subjects exceeded that expected by chance $[t(6)=$ $30.00, \mathrm{p}<.0001]$.

$\mathbf{A} \rightarrow \mathbf{B} \rightarrow \mathbf{C} \rightarrow \mathbf{D}$ training. The proportion of trials completed correctly during $A \rightarrow B \rightarrow C \rightarrow D$ simultaneous training is shown in the bottom panel of Figure 1. Five of the seven subjects trained on $A B C D$ arrays met the criterion of $70 \%$ accuracy. (The accuracy of Subjects 203 and 299 reached asymptotic levels of approximately $35 \%$ after 54 and 66 sessions, ' respectively, of training.)

When repeat responses are allowed, chance performance is less than $1 \%$. This can be calculated as follows: $p$ (correct $A$ response) $=1 / 4=.25$. After a correct first choice, repeats to $B$ and $C$ are allowed and, after a correct second choice, repeats to $C$ are allowed. Thus, the probability of correct $B, C$, and $D$ responses $=1 / 3=.33 ; \quad p(A \rightarrow B \rightarrow C \rightarrow D$ sequence $)=$ $.25 \times .33 \times .33 \times .33=.0098$. The performance of all subjects trained on $A \rightarrow B \rightarrow C \rightarrow D$ arrays exceeded chance levels of accuracy at the end of training $[70 \%$ birds, $\mathrm{t}(4)=50.32, \mathrm{p}<.0005 ; 35 \%$ birds, $\mathrm{t}(1)=97.04$, $\mathrm{p}<.005$ ].

In interpreting the performance of our subjects, it is of interest to know whether they learned the sequences $A \rightarrow B \rightarrow C$ and $A \rightarrow B \rightarrow C \rightarrow D$ as general concepts or whether their performance was specific to the 80 training arrays used during three- and fourelement training. Accordingly, we will consider the results of the $A \rightarrow B \rightarrow C$ and $A \rightarrow B \rightarrow C \rightarrow D$ generalization tests before considering details of performance during training. Analyses of the performance of the subjects of Groups 1 and 2 during all phases of training, including generalization testing, failed to yield any significant differences. Accordingly, the data from these groups will be collapsed in subsequent analyses.

\section{Generalization to Novel Arrays}

Figure 2 summarizes the results of all of the generalization tests based upon three- and four-color sequences. Performance is averaged across all subjects. For each sequence, generalization from training to novel arrays was virtually complete. It was also the case that latencies to different elements of the novel 


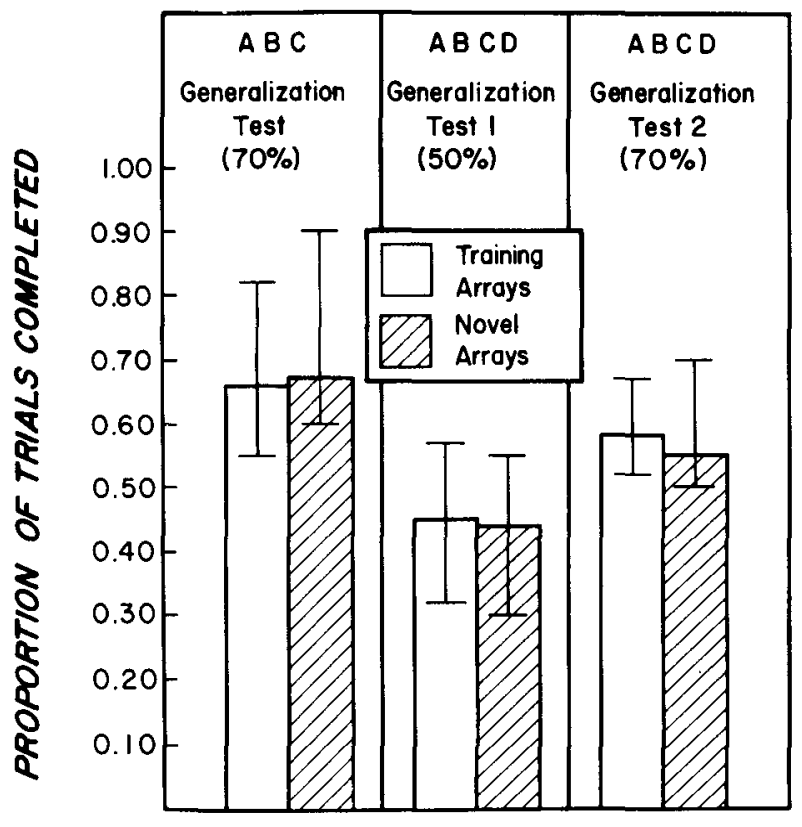

TYPE OF ARRAY

Figure 2. Average proportion of training and novel arrays completed during the $A \rightarrow B \rightarrow C$ generalization test at $70 \%$ accuracy (left-hand panel), and during the $A \rightarrow B \rightarrow C \rightarrow D$ generalization tests at $50 \%$ (middle panel) and $70 \%$ accuracy (right-hand panel).

arrays were indistinguishable from latencies to the elements of the training arrays (see below, bottom portions of Tables 4 and 5).

Not shown in Figure 2 is the immediacy of generalization to the novel arrays. During the $A \rightarrow B \rightarrow C$ test, an average of $60.0 \%$ of novel arrays were completed correctly during the first five novel array trials, as compared with $54.29 \%$ of the first five training arrays $[\mathrm{t}(6)=1.99, \mathrm{p}>.05]$. During the $50 \% \mathrm{~A} \rightarrow \mathrm{B} \rightarrow$ $\mathrm{C} \rightarrow \mathrm{D}$ test, $37.14 \%$ of the first five novel arrays and $34.29 \%$ of the first five training arrays were completed correctly $[\mathrm{t}(6)=.33, \mathrm{p}>.5]$. During the $70 \%$ test, an average of $56.00 \%$ of the first five novel arrays, and $40.00 \%$ of the first five training arrays, were completed correctly $[t(4)=1.63, p>.10]$. Even though there was an overall decrement in the proportion of trials completed during the generalization tests (compared with the relevant training baselines), accuracy of responding to the novel and the training arrays could not be distinguished. Such fluctuations in accuracy fall within the range of session-to-session variation observed in Figure 1.

\section{Repeat Pecks, Errors, and Latencies}

The upper portion of Figure 3 summarizes the distribution of responses at each position of the sequence during the two sessions in which $70 \%$ of trials were completed correctly. The proportion of correct responses decreased, and the proportion of repeat responses increased, as the subject progressed through the sequence. This was true during both $A \rightarrow B \rightarrow C$ and $A \rightarrow B \rightarrow C \rightarrow D$ training. However, the sum of the proportion of correct and repeat responses at each position of the sequence was fairly constant (see also Tables 4 and 5 below).

Of all errors, forward errors (defined as pecking $B$ or $C$ at trial onset, or the sequence $A \rightarrow C$ ) were much more prevalent than backward errors $(90.48 \%$ vs. $9.52 \%$ of all errors during $A \rightarrow B \rightarrow C$ training and $88.67 \%$ vs. $11.32 \%$ of all errors during $A \rightarrow B \rightarrow C \rightarrow D$ training. At the end of $A \rightarrow B \rightarrow C$ training, $97.77 \%$ of the forward errors were one-skip errors, whereas only $2.33 \%$ were two-skip errors. At the end of $\mathrm{A} \rightarrow \mathrm{B} \rightarrow$ $C \rightarrow D$ training, $94.44 \%$ of all forward errors were one-skip errors, $5.0 \%$ were two-skip errors, and $56 \%$ were three-skip errors.

Figure 4 summarizes repetitive responding during training 'on two-, three-, and four-color sequences. Each panel shows the mean and the range of the number of repeats that occurred prior to correct and incorrect transitions during the $70 \%$ criterion sessions at each stage of training. In every instance in which forward errors were possible, there were at least as many repeat responses prior to a forward error than prior to a correct response. Overall, there was no difference in the average number of repeats prior to correct and incorrect transitions of criterion responding during $A \rightarrow B \rightarrow C$ and $A \rightarrow B \rightarrow C \rightarrow D$ training $[t(6)=1.15, p>.10]$. Whatever the function of repeat responses, there is no evidence that they serve to increase accuracy. A similar picture can be seen in Table 2, which presents the average time spent on repetitive responding for correct and incorrect transi-

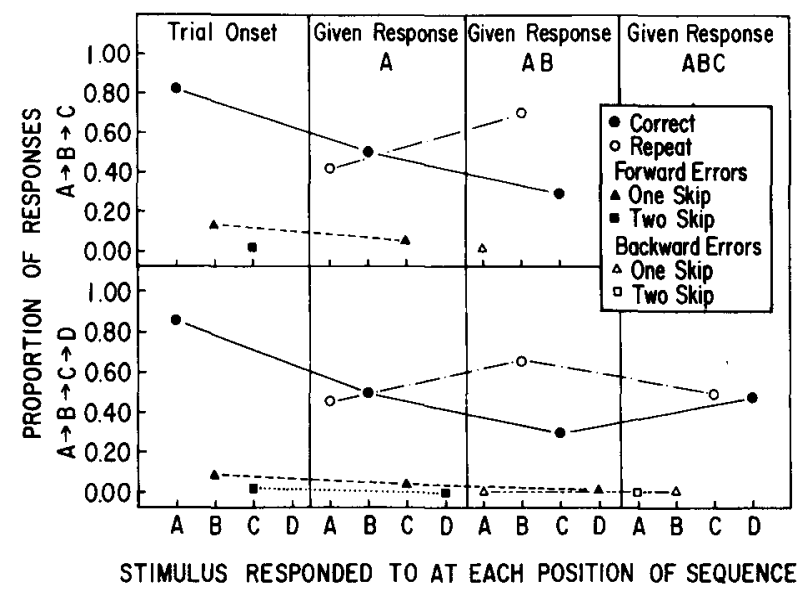

Figure 3. Proportion of all correct, incorrect, and repetitive responses at a given position of the $A \rightarrow B \rightarrow C$ (upper panel) and $A \rightarrow B \rightarrow C \rightarrow D$ (lower panel) sequences. The types of errors refer to responses to colors that are removed (skips) from the correct color towards the beginning of the sequence (backward) or towards the final color of the sequence (forward). 


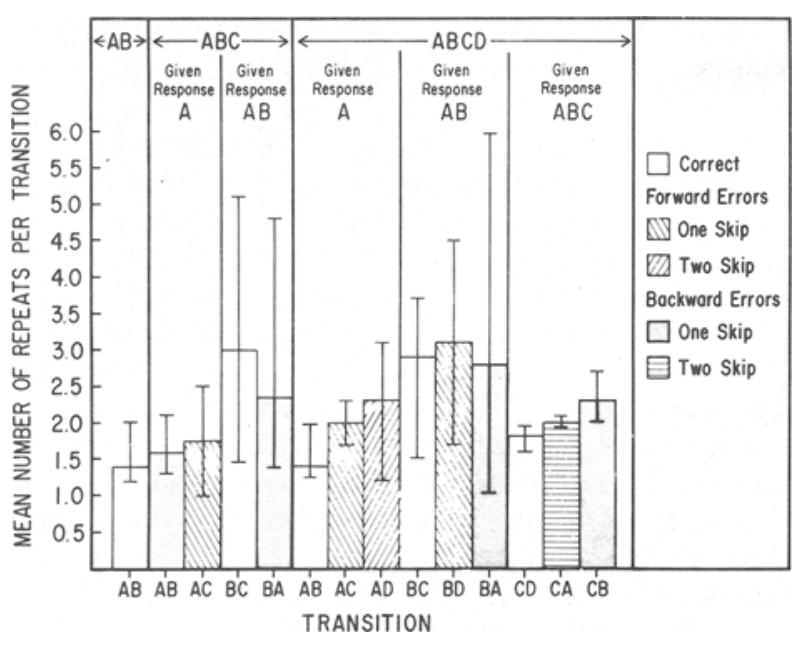

Figure 4. Average number of repetitive responses to a given stimulus prior to correct and incorrect responses at three stages of training: $A \rightarrow B$ only, $A \rightarrow B \rightarrow C$ only, and $A \rightarrow B \rightarrow C \rightarrow D$. Vertical lines indicate range of each distribution. The types of errors refer to responses to colors that are removed from the correct color (skips) towards the beginning of the sequence (backward) or towards the final color of the sequence (forward).

tions during $A \rightarrow B \rightarrow C \rightarrow D$ training. At each position of the sequence, at least as much time was spent on repeat responses prior to an error as was spent on repeat responses prior to a correct response.

The upper portion of Figure 5 presents the average values and the ranges of the latencies of correct, incorrect, and repeat responses at each position of the sequence $A \rightarrow B \rightarrow C$ (for the sessions during which the $70 \%$ criterion was met). The shortest latencies occurred in the case of repeat pecks. The mean latency of correct pecks across all positions of the sequence

Table 2

Average Time (in Seconds) Spent on Repeats During $A \rightarrow B \rightarrow C$ and $A \rightarrow B \rightarrow C \rightarrow D$ Terminal Performance

\begin{tabular}{|c|c|c|}
\hline Transition & $A \rightarrow B \rightarrow C$ & $\mathrm{~A} \rightarrow \mathrm{B} \rightarrow \mathrm{C} \rightarrow \mathrm{D}$ \\
\hline & \multicolumn{2}{|c|}{ Correct } \\
\hline $\mathrm{A} \rightarrow \mathrm{B}$ & .20 & .26 \\
\hline $\mathrm{AB} \rightarrow \mathrm{C}$ & .78 & .58 \\
\hline \multirow[t]{2}{*}{$\mathrm{ABC} \rightarrow \mathrm{D}$} & & .39 \\
\hline & \multicolumn{2}{|c|}{ Forward Errors } \\
\hline \multicolumn{3}{|l|}{ One-Skip } \\
\hline$A \rightarrow C$ & .36 & .56 \\
\hline $\mathrm{AB} \rightarrow \mathrm{D}$ & & .93 \\
\hline \multicolumn{3}{|l|}{ Two-Skip } \\
\hline \multirow[t]{2}{*}{$A \rightarrow D$} & & .65 \\
\hline & \multicolumn{2}{|c|}{ Backward Errors } \\
\hline \multicolumn{3}{|l|}{ One-Skip } \\
\hline $\mathrm{AB} \rightarrow \mathrm{A}$ & .75 & .44 \\
\hline $\mathrm{ABC} \rightarrow \mathrm{B}$ & & .56 \\
\hline \multicolumn{3}{|l|}{ Two-Skip } \\
\hline $\mathrm{ABC} \rightarrow \mathrm{A}$ & & .10 \\
\hline
\end{tabular}

was $1.03 \mathrm{sec}$, of forward one-skip errors, $1.85 \mathrm{sec}$, and of forward two-skip errors, $2.31 \mathrm{sec}$.

Extensive observation of the behavior of our subjects suggested that some forward errors result from incomplete pecks, or "air" pecks. An air peck is directed at the response key, but it either falls short or is of insufficient force to activate the microswitch. That some forward errors result from the sequence of an air peck and a peck to a downstream color is suggested by the systematic increase in the latency of forward errors as the number of "skips" beyond the correct color increases.

The lower portion of Figure 5 shows mean peck latencies at each position in the sequence $A \rightarrow B \rightarrow C \rightarrow D$ during the $70 \%$ criterion sessions. The latency of firstposition forward one-skip errors was $1.68 \mathrm{sec}, 1.7$ times as long as the latency of correct $A$ responses $(.98 \mathrm{sec})$. The latency of second-position forward one-skip errors $(\mathrm{A} \rightarrow \mathrm{C})$ was $1.13 \mathrm{sec}, 1.5$ times as long as the latency of correct $B$ responses $(.75 \mathrm{sec})$. Forward two- and three-skip errors (C, and D at trial onset; $A \rightarrow D$ ) were quite infrequent. They accounted for only $4 \%$ of all responses at each position. The latencies of such errors tended, however, to be longer than the latencies of correct responses and one-skip errors.

\section{Performance as a Function of Position in the Sequence}

The average relative frequencies and latencies of responses at each position of the sequence during $A \rightarrow B, A \rightarrow B \rightarrow C$, and $A \rightarrow B \rightarrow C \rightarrow D$ training are summarized in Tables 3, 4, and 5, respectively. Each row represents performance at a given position in the sequence, Tables 4 and 5 show data averaged across two sessions per bird at each of three levels of performance accuracy: $25 \%, 50 \%$, and $70 \%$ accuracy. Also shown is generalization test performance to both training and novel arrays.

The major left-right diagonal indicates the relative frequencies and latencies of all correct responses at a given position of the sequence. Repetitive pecking is shown in the left-right minor diagonal immediately below the major diagonal. Forward errors are shown in the minor diagonals above the major diagonal, and backward errors, by the minor diagonals below the diagonal showing repetitive pecks. The bottom rows of each table indicate accuracy to each color when repetitive responses are excluded.

$\mathbf{A} \rightarrow \mathbf{B}$ training. When repeat pecks to $\mathbf{A}$ were included in calculating the percentage of pecks to $B$ (immediately after an $\mathbf{A}$ peck), the average percentage of pecks to B was 58.28 (cf. Table 3, first row). After the initial peck, $41.72 \%$ of all pecks to A were repeat pecks to $A$. When repeat pecks are not considered, $99.34 \%$ of pecks following the initial peck to A occurred to B. (The deviation from a propor- 


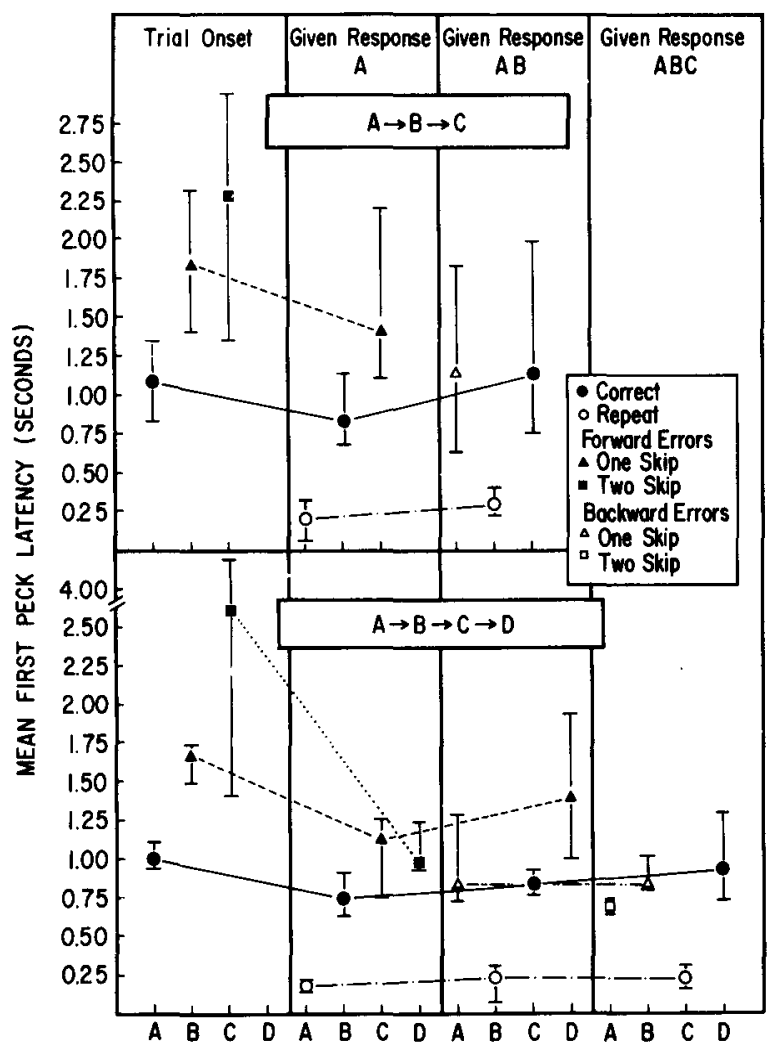

STIMULUS RESPONDED TO AT EACH POSITION OF SEQUENCE

Figure 5. Average latency of responding to correct, incorrect, and repetitive colors at each position of the $A \rightarrow B \rightarrow C$ and $A \rightarrow B \rightarrow$ $C \rightarrow D$ sequences at $70 \%$ accuracy.

tion of 1.00 was due to trials that terminated because $15 \mathrm{sec}$ had elapsed without a peck to $B$.)

Forward errors (responses to $B$ at trial onset) accounted for $19.48 \%$ of all responses in the first position of the sequence. The latency of forward errors was almost twice as long $(2.19 \mathrm{sec})$ as the latency of correct responses to $\mathrm{A}(1.13 \mathrm{sec})$. The difference between the average latency of $B$ errors and correct A responses $(1.06 \mathrm{sec})$ is strikingly close to the average latency of correct $B$ responses at the second position of the sequence (mean $=1.08 \mathrm{sec}$ ) and is consistent

Table 3

Relative Frequency (F) and Latency (L) of Responses at Each Position of AB Arrays

\begin{tabular}{|c|c|c|c|c|c|}
\hline \multirow{3}{*}{$\begin{array}{c}\text { Correct } \\
\text { Stimulus }\end{array}$} & \multirow[b]{3}{*}{ Pecks } & \multicolumn{4}{|c|}{ Stimulus Responded to } \\
\hline & & \multicolumn{2}{|c|}{ A } & \multicolumn{2}{|c|}{$\mathrm{B}$} \\
\hline & & $\mathbf{F}$ & $\mathbf{L}$ & $\mathbf{F}$ & $\mathbf{L}$ \\
\hline $\begin{array}{l}\text { A } \\
\text { B }\end{array}$ & $\begin{array}{l}1237 \\
1697\end{array}$ & $\begin{array}{l}80.52 \\
41.72\end{array}$ & $\begin{array}{r}1.13 \\
.68\end{array}$ & $\begin{array}{l}19.48 \\
58.28\end{array}$ & $\begin{array}{l}2.19 \\
1.08\end{array}$ \\
\hline PC & & 80.52 & & 99.34 & \\
\hline
\end{tabular}

Note-PC = percent correct without repeats. Frequencies are given in percentages; latencies are given in seconds. with the previously mentioned possibility that air pecks to an intervening element are responsible for many forward errors.

$\mathbf{A} \rightarrow \mathbf{B} \rightarrow \mathbf{C}$ training. In Table 3 , the rows summarizing performance at $25 \%$ accuracy shows why the percentage of correct responses to A dropped from $80.52 \%$ to $69.56 \%$ as the sequence was lengthened from two to three colors. At $25 \%$ accuracy, the level of incorrect pecks to $\mathrm{C}$ (forward two-step errors) was $15.13 \%$. At $70 \%$ accuracy, the level of such errors decreased to $2.39 \%$. A similar decrease in one-skip errors can be seen at the second position of the sequence. These data support the hypothesis that, at this stage of training $(A \rightarrow B)$, choice of the second color in the sequence is by default and simply involves a discrimination of "what hasn't been pecked."

$\mathbf{A} \rightarrow \mathbf{B} \rightarrow \mathbf{C} \rightarrow \mathbf{D}$ training. A comparison of the frequencies of correct responding (without repeats) at the end of $A \rightarrow B \rightarrow C$ training (Table 4, bottom) with initial performance on $A \rightarrow B \rightarrow C \rightarrow D$ arrays shows that much of the decrement in accuracy at the beginning of $A \rightarrow B \rightarrow C \rightarrow D$ training could be attributed to forward errors. At the beginning of $A \rightarrow B \rightarrow C \rightarrow D$ training, accuracy of responding to $A$ dropped from $82.81 \%$ to $79.73 \%$. Accuracy of responding at the $B$ and $C$ positions dropped from $87.45 \%$ to $75.56 \%$ (B) and from $95.37 \%$ to $64.07 \%$ (C).

Serial position effect. Figure 6 shows the proportion of correct responses at each position of the $A \rightarrow B \rightarrow C$ sequence. Separate accuracy functions are presented at each of three levels of performance (completion of $25 \%, 50 \%$, and $70 \%$ of trials). A U-shaped function was obtained at accuracy levels of $25 \%$. In the case of $A \rightarrow B \rightarrow C$ training, there was no significant relation between overall accuracy and serial position $[F(2,10)=3.67, p>.05]$. During $A \rightarrow B \rightarrow C \rightarrow D$ training, a significant position effect was obtained at the $25 \%$ accuracy level $(\mathrm{N}=7) \quad[\mathrm{F}(3,15)=4.95$, $\mathrm{p}<.025]$ with a significant quadratic trend $[\mathrm{F}(1,5)=$ $7.71, \mathrm{p}<.05]$.

By the time the $70 \%$ criterion was satisfied $(\mathrm{N}=5)$ on both $A \rightarrow B \rightarrow C$ and on $A \rightarrow B \rightarrow C \rightarrow D$ arrays, the proportion of correct transitions increased monotonically with position in the sequence. On $A \rightarrow B \rightarrow C$ arrays, there was an overall effect of position on accuracy $[F(2,10)=15.59, p<.001]$ characterized by a significant linear trend across subjects $[F(1,5)=47.08$, $\mathrm{p}<.001$ ]. On $\mathrm{A} \rightarrow \mathrm{B} \rightarrow \mathrm{C} \rightarrow \mathrm{D}$ arrays, there was a significant position effect $[F(3,9)=14.23, p<.001]$ with a significant linear trend $[F(1,3)=71.67$, $\mathrm{p}<.005]$.

\section{EXPERIMENT 2}

The second experiment asked whether birds trained on four-color arrays $(A \rightarrow B \rightarrow C \rightarrow D)$ could perform the sequence when one or more adjacent colors (e.g., 
Table 4

Relative Frequency (F) and Latency (L) of Responses at Each Position of ABC Arrays

\begin{tabular}{|c|c|c|c|c|c|c|c|}
\hline \multirow[b]{3}{*}{ Condition } & \multirow[b]{3}{*}{ Pecks } & \multicolumn{6}{|c|}{ Stimulus Responded to } \\
\hline & & \multicolumn{2}{|c|}{ A } & \multicolumn{2}{|c|}{$\mathrm{B}$} & \multicolumn{2}{|c|}{$\mathrm{C}$} \\
\hline & & $\mathrm{F}$ & $\mathbf{L}$ & $\mathbf{F}$ & $\mathrm{L}$ & $\mathbf{F}$ & $\mathbf{L}$ \\
\hline & \multicolumn{7}{|c|}{ Correct Stimulus: A } \\
\hline $25 \%$ & 1104 & 69.56 & 1.225 & 15.31 & 2.281 & 15.13 & 2.337 \\
\hline $50 \%$ & 1082 & 76.25 & 1.135 & 18.67 & 2.093 & 5.08 & 3.111 \\
\hline $70 \%$ & 1088 & 82.81 & 1.093 & 14.80 & 1.841 & 2.39 & 2.315 \\
\hline GT(T) & 414 & 80.91 & 1.111 & 14.98 & 1.744 & 4.10 & 2.105 \\
\hline \multirow[t]{2}{*}{ GT(N) } & 140 & 79.29 & 1.182 & 17.14 & 1.930 & 3.57 & 2.533 \\
\hline & \multicolumn{7}{|c|}{ Correct Stimulus : B } \\
\hline $25 \%$ & 1435 & 47.52 & .306 & 33.38 & 1.108 & 19.09 & 1.422 \\
\hline $50 \%$ & 1602 & 48.88 & .232 & 41.26 & .974 & 9.86 & 1.687 \\
\hline $70 \%$ & 1553 & 43.01 & .202 & 50.67 & .867 & 6.32 & 1.418 \\
\hline $\mathrm{GT}(\mathrm{T})$ & 522 & 36.97 & .183 & 54.79 & .882 & 8.24 & 1.647 \\
\hline \multirow[t]{2}{*}{ GT(N) } & 188 & 41.49 & .266 & 51.60 & .851 & 6.91 & 1.613 \\
\hline & \multicolumn{7}{|c|}{ Correct Stimulus: $C$} \\
\hline $25 \%$ & 1369 & 8.40 & .975 & 66.03 & 1.918 & 25.56 & 1.069 \\
\hline $50 \%$ & 2400 & 2.25 & 1.071 & 72.96 & .327 & 24.79 & 1.053 \\
\hline $70 \%$ & 2532 & 1.18 & 1.136 & 69.19 & .311 & 29.62 & 1.167 \\
\hline GT(T) & 842 & 1.42 & 1.041 & 66.39 & .276 & 32.19 & 1.164 \\
\hline \multirow[t]{2}{*}{ GT(N) } & 318 & .94 & 1.027 & 70.13 & .295 & 28.93 & .878 \\
\hline & \multicolumn{7}{|c|}{ Percent Correct Without Repeats } \\
\hline $25 \%$ & & 69.56 & & 62.59 & & 74.19 & \\
\hline $50 \%$ & & 76.25 & & 80.04 & & 90.15 & \\
\hline $70 \%$ & & 82.81 & & 87.45 & & 95.37 & \\
\hline $\mathrm{GT}(\mathrm{T})$ & & 80.91 & & 84.84 & & 94.84 & \\
\hline $\mathrm{GT}(\mathrm{N})$ & & 79.29 & & 87.13 & & 94.77 & \\
\hline
\end{tabular}

Note-Frequencies are given in percentages; latencies are given in seconds.

B or C) were omitted or when the sequence had to be started at a color other than A (e.g., B or C). Having learned the sequence $A \rightarrow B \rightarrow C \rightarrow D$, a pigeon that responded accurately to a subsequence such as BD would demonstrate that it had learned about relationships between nonadjacent as well as adjacent elements of the sequence. Accurate performance on such subsequences cannot be accounted for by an associative chaining or S-R model, a model which assumes that subjects learn only forward associations between adjacent items in the sequence.

As mentioned earlier, one line of evidence that questions the adequacy of the S-R model of chaining as an explanation of serial behavior in humans is the failure of such models to account for sentence production or comprehension (Chomsky, 1957, 1959; Lashley, 1951). In defense of the adequacy of chaining theory, it might be argued that remote associations may exist between nonadjacent elements and that such associations are mediated by the meaning of the elements. In learning lists of words or phrases of a sentence, or even elements of a list of trigrams, human subjects typically assign meanings or interpretations to items that have little or no inherent meaning. Since there is no basis for assuming that a pigeon would encode disparate elements of a sequence, it is of interest to ask whether remote associations can be demonstrated following the acquisition of the $A \rightarrow B \rightarrow C \rightarrow D$ sequence trained in Experiment 1.

\section{Method}

\section{Subjects}

The subjects were the seven White Carneaux pigeons from the first experiment.

\section{Apparatus}

The apparatus was the same as that used in Experiment 1. Test arrays consisting of two- and three-color subsets of the original four colors were substituted for the four-color arrays. The test stimuli were prepared in the same manner as in Experiment 1.

\section{Procedure}

Following generalization testing with $A \rightarrow B \rightarrow C \rightarrow D$ arrays, each of the seven subjects was tested with arrays of two or three colors. In order to evaluate the influence of accuracy of performance on $A \rightarrow B \rightarrow C \rightarrow D$ arrays on generalization to subsequences, Subjects 203 and 296 of Group 2 were tested after completing the $A \rightarrow B \rightarrow$ $\mathrm{C} \rightarrow \mathrm{D}$ generalization test given after the $50 \%$ accuracy criterion was satisfied. Subjects $262,263,259,298$, and 299 were tested following the generalization test given after the $70 \%$ accuracy criterion was satisfied.

Each two- and three-color test consisted of two consecutive 80-trial sessions in which all possible two(or three-)color combinations of the colors A, B, C, and D were presented. Correct trials consisted of pecking the colors of each subsequence in the 
Table 5

Relative Frequency (F) and Latency (L) of Responses at Each Position of ABCD Arrays

\begin{tabular}{|c|c|c|c|c|c|c|c|c|c|}
\hline \multirow[b]{3}{*}{ Condition } & \multirow[b]{3}{*}{ Pecks } & \multicolumn{8}{|c|}{ Stimulus Responded to } \\
\hline & & \multicolumn{2}{|c|}{$\mathbf{A}$} & \multicolumn{2}{|c|}{ B } & \multicolumn{2}{|c|}{$\mathrm{C}$} & \multicolumn{2}{|c|}{$\mathrm{D}$} \\
\hline & & $\mathbf{F}$ & $\mathbf{L}$ & $\mathrm{F}$ & $\mathbf{L}$ & $\mathbf{F}$ & $\mathbf{L}$ & $\mathbf{F}$ & $\mathbf{L}$ \\
\hline & \multicolumn{9}{|c|}{ Correct Stimulus : A } \\
\hline $25 \%$ & 1110 & 79.73 & 1.187 & 15.58 & 1.918 & 3.24 & 3.014 & 1.44 & 3.741 \\
\hline $50 \%$ & 1114 & 84.65 & 1.082 & 13.02 & 1.994 & 1.88 & 1.802 & .45 & 3.100 \\
\hline GT(T) & 415 & 76.39 & 1.136 & 16.39 & 2.115 & 6.75 & 2.492 & .47 & 1.400 \\
\hline GT(N) & 140 & 70.72 & 1.103 & 22.14 & 1.862 & 6.43 & 2.646 & .71 & 2.800 \\
\hline $70 \%$ & 794 & 85.64 & .990 & 11.21 & 1.650 & 3.15 & 2.844 & .00 & .000 \\
\hline GT(T) & 298 & 78.19 & 1.001 & 16.44 & 1.795 & 4.70 & 1.727 & .67 & 3.992 \\
\hline \multirow[t]{2}{*}{ GT(N) } & 100 & 83.00 & 1.046 & 15.00 & 1.830 & 2.00 & 1.300 & .00 & .000 \\
\hline & \multicolumn{9}{|c|}{ Correct Stimulus : B } \\
\hline $25 \%$ & 1537 & 42.49 & .211 & 43.46 & .884 & 8.65 & 1.779 & 5.40 & 1.975 \\
\hline $50 \%$ & 1504 & 37.50 & .216 & 53.12 & .805 & 6.32 & 1.383 & 3.06 & 1.557 \\
\hline $\mathrm{GT}(\mathrm{T})$ & 529 & 40.27 & .164 & 49.53 & .820 & 7.75 & 1.386 & 2.46 & 1.423 \\
\hline GT(N) & 170 & 41.76 & .155 & 46.47 & .863 & 7.65 & 1.831 & 4.12 & 1.618 \\
\hline $70 \%$ & 1212 & 44.06 & .186 & 50.25 & .743 & 4.45 & 1.142 & 1.24 & .973 \\
\hline GT(T) & 375 & 37.87 & .189 & 53.33 & .776 & 6.40 & 1.232 & 2.40 & 1.131 \\
\hline \multirow[t]{2}{*}{$\mathrm{GT}(\mathrm{N})$} & 139 & 41.01 & .274 & 46.76 & .729 & 7.19 & 1.845 & 5.04 & 1.345 \\
\hline & \multicolumn{9}{|c|}{ Correct Stimulus: C } \\
\hline $25 \%$ & 1968 & 1.27 & .958 & 66.21 & .239 & 21.65 & 1.155 & 10.87 & 1.558 \\
\hline $50 \%$ & 2331 & 1.54 & 1.084 & 65.81 & .227 & 27.33 & .939 & 5.32 & 1.620 \\
\hline $\mathrm{GT}(\mathrm{T})$ & 728 & 1.10 & 1.051 & 64.01 & .223 & 29.12 & .991 & 5.77 & 1.773 \\
\hline GT(N) & 233 & 1.71 & .775 & 66.09 & .226 & 28.33 & .943 & 3.86 & 1.570 \\
\hline $70 \%$ & 1816 & .88 & .829 & 66.47 & .214 & 29.79 & .798 & 2.86 & 1.434 \\
\hline $\mathrm{GT}(\mathrm{T})$ & 558 & 1.25 & 1.207 & 64.15 & .238 & 30.11 & .826 & 4.48 & 1.395 \\
\hline \multirow[t]{2}{*}{ GT(N) } & 195 & .00 & .000 & 66.67 & .288 & 29.23 & .842 & 4.10 & 1.010 \\
\hline & \multicolumn{9}{|c|}{ Correct Stimulus: D } \\
\hline $25 \%$ & 806 & .50 & 1.247 & 9.93 & .965 & 46.70 & .223 & 41.56 & 1.200 \\
\hline $50 \%$ & 1310 & .00 & .000 & 3.97 & 1.184 & 51.83 & .292 & 44.20 & 1.098 \\
\hline GT(T) & 450 & .00 & .000 & 4.67 & .852 & 53.11 & .271 & 42.22 & 1.181 \\
\hline GT(N) & 127 & .00 & .000 & 4.73 & .927 & 48.03 & .247 & 47.24 & 1.180 \\
\hline $70 \%$ & 1077 & .09 & .700 & 1.21 & .856 & 49.77 & .227 & 48.93 & .941 \\
\hline $\mathrm{GT}(\mathrm{T})$ & 309 & .00 & .000 & 1.94 & .683 & 45.63 & .232 & 52.43 & 1.032 \\
\hline \multirow[t]{2}{*}{ GT(N) } & 155 & .00 & .000 & 3.22 & .600 & 63.87 & .330 & 32.90 & 1.025 \\
\hline & \multicolumn{9}{|c|}{ Percent Correct Without Repeats } \\
\hline $25 \%$ & & 79.73 & & 75.38 & & 63.77 & & 78.31 & \\
\hline $50 \%$ & & 84.65 & & 84.88 & & 79.45 & & 90.93 & \\
\hline GT(T) & & 76.39 & & 82.65 & & 80.47 & & 89.55 & \\
\hline GT(N) & & 70.72 & & 79.93 & & 83.26 & & 90.10 & \\
\hline $70 \%$ & & 85.64 & & 89.42 & & 89.01 & & 97.40 & \\
\hline $\mathrm{GT}(\mathrm{T})$ & & 78.19 & & 85.87 & & 84.01 & & 96.66 & \\
\hline GT(N) & & 83.00 & & 77.60 & & 88.84 & & 90.23 & \\
\hline
\end{tabular}

Note-Frequencies are given in percentages; latencies are given in seconds.

order consistent with the trained $A \rightarrow B \rightarrow C \rightarrow D$ sequence. As in Experiment 1, a correct sequence of pecks terminated the trial with reinforcement. Errors also terminated the trial and resulted in a 4-sec time-out during which the chamber was darkened. Between trials; a variable 15-sec ITI was in effect. All trials were terminated if they were not completed within $15 \mathrm{sec}$.

The two-color test consisted of the subsequences $A \rightarrow B, A \rightarrow C$, $A \rightarrow D, B \rightarrow C, B \rightarrow D$ and $C \rightarrow D . A \rightarrow D$ and $B \rightarrow C$ arrays were presented 14 times during each of the two successive test sessions. The remaining arrays were each presented 13 times per session. The arrays presented were selected randomly from all of the possible configurations of two colors on six response keys. During the two three-color test sessions, each of the following arrays was presented 20 times per session: $A \rightarrow B \rightarrow C, A \rightarrow B \rightarrow D, A \rightarrow C \rightarrow D$, and $B \rightarrow C \rightarrow D$. The arrays presented were selected randomly from all of the possible configurations of three colors on six response keys.
Group 1 Subjects 262 and 263 and Group 2 Subject 203 were first tested with two-color subsequences. Following baseline training with $A \rightarrow B \rightarrow C \rightarrow D$ (one session at $70 \%$ accuracy), they were tested with three-color arrays. Group 1 Subject 259 and Group 2 Subjects 298, 299, and 296 were tested first with three-color subsequences and then, after four-color baseline training (one session at $70 \%$ accuracy), with the two-color arrays.

\section{Results}

\section{Two-Color Subsequence Test}

No differences could be detected between the performance of subjects who were tested first with tworather than three-color subsequences. Accordingly, 


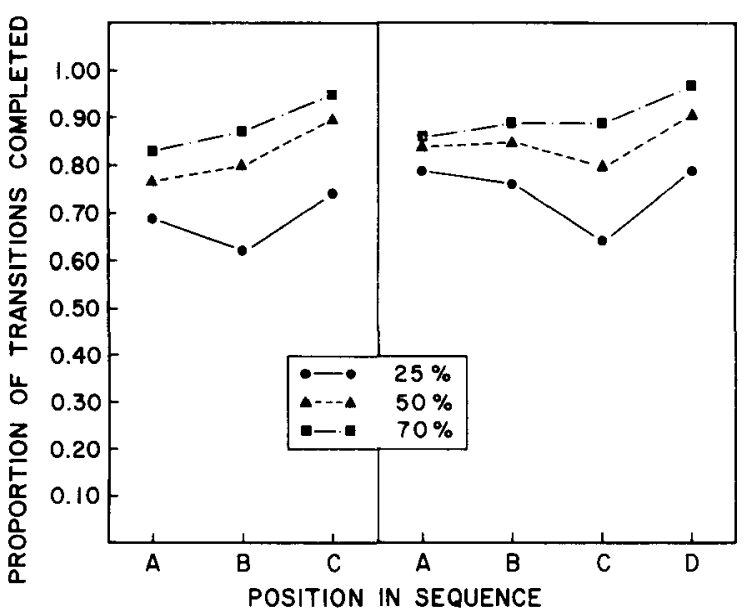

Figure 6. Proportion of transitions correctly completed at each position of the $A \rightarrow B \rightarrow C$ (left-hand panel) and $A \rightarrow B \rightarrow C \rightarrow D$ (righthand panel) sequence at three levels of training accuracy: completion of $25 \%, 50 \%$, and $70 \%$ of trials.

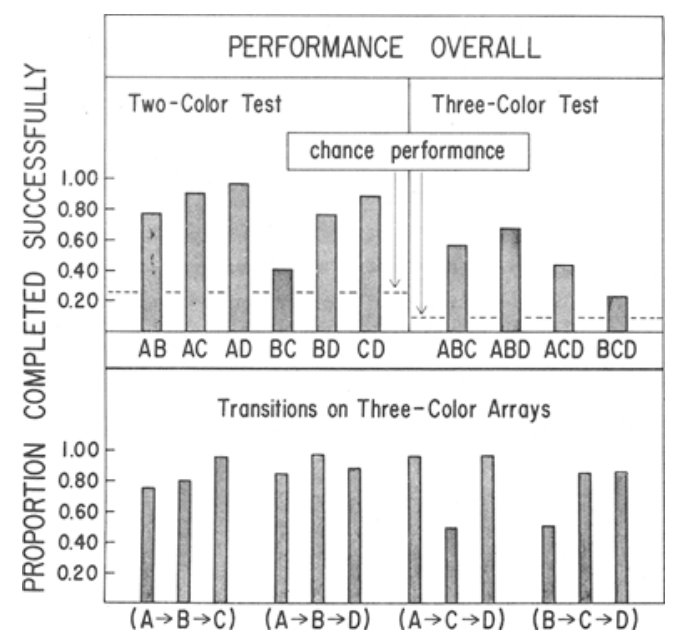

Figure 7. Upper panel: Proportion of trials completed correctly for each two- and three-color subsequence. Lower panel: Proportion of correct transitions on three-color subsequences.

results from all two-color tests will be presented together.

The average proportion of trials correctly completed to each subsequence during the first five trials with each was as follows: $A \rightarrow B, 77.14 ; A \rightarrow C, 91.43$; $A \rightarrow D, 94.29 ; B \rightarrow C, 37.14 ; B \rightarrow D, 68.37 ; C \rightarrow D, 80.00$. Thus, accuracy of responding to five of the six twocolor subsequences exceeded $50 \%$, the value predicted by a model of random choice of colors $[\mathrm{t}(5)=$ $2.92, p<.025]$. Figure 7 (upper left-hand panel) presents the proportion and range of trials completed correctly for each subsequence during the first test session. While performance did not differ during the second test session, it was not included on the grounds that some learning may have occurred during the first test session. Averaged across subjects, the six subsequences ranked by decreasing proportion of trials completed during the first test session were: $A \rightarrow D$, 94.35\%; $A \rightarrow C, 87.78 \% ; C \rightarrow D, 86.50 \% ; B \rightarrow D$, $75.00 \% ; A \rightarrow B, 74.76 \%$; and $B \rightarrow C, 38.70 \%$.

The poor performance to the $B \rightarrow C$ subsequence (38.70\%) is puzzling. At the end of $A \rightarrow B \rightarrow C \rightarrow D$ training, color $B$ was selected at position $B$ on $89.42 \%$ of the trials (repeat responses excluded). This indicates that the difficulty with $B \rightarrow C$ subsequences was not due to a failure to discriminate $B$ (red) from $C$ (green).

Accuracy of responding to $A$ and $B$, as first choice colors, increased with increasing separation in the original $A \rightarrow B \rightarrow C \rightarrow D$ sequence of the second color from the first $[F(2,12)=5.36, p<.002]$. Accuracy to stimulus $A$ increased from $74.46 \%$ on $A \rightarrow B$ arrays to $87.78 \%$ on $A \rightarrow C$ arrays, to $94.35 \%$ on $A \rightarrow D$ arrays. Responding to $B \rightarrow D$ arrays was more accurate than responding to $\mathrm{B} \rightarrow \mathrm{C}$ arrays $(75.46 \%$ vs. $38.70 \%)$.

The upper portion of Figure 8 shows the average latencies of first pecks to both colors for each twocolor subsequence. The latency of first-stimulus pecks to subsequences beginning with $B$ or $C$ was significantly longer than the latencies of pecks to subsequences beginning with $\mathrm{A}[\mathrm{t}(6)=2.88, \mathrm{p}<.005]$. Not shown in Figure 8 is the striking difference between the latencies to A and B, as first element stimuli, after testing at $50 \%$ accuracy during $A \rightarrow B \rightarrow C \rightarrow D$ training. At the $50 \%$ level of accuracy, the mean latencies of responses to $\mathrm{A}$ and $\mathrm{B}$ were 1.082 and $.805 \mathrm{sec}$, respectively. The mean latencies of responding to $A$ and to $B$ of subjects tested after satisfying the $70 \%$ criterion were 1.01 and $1.32 \mathrm{sec}$, re-

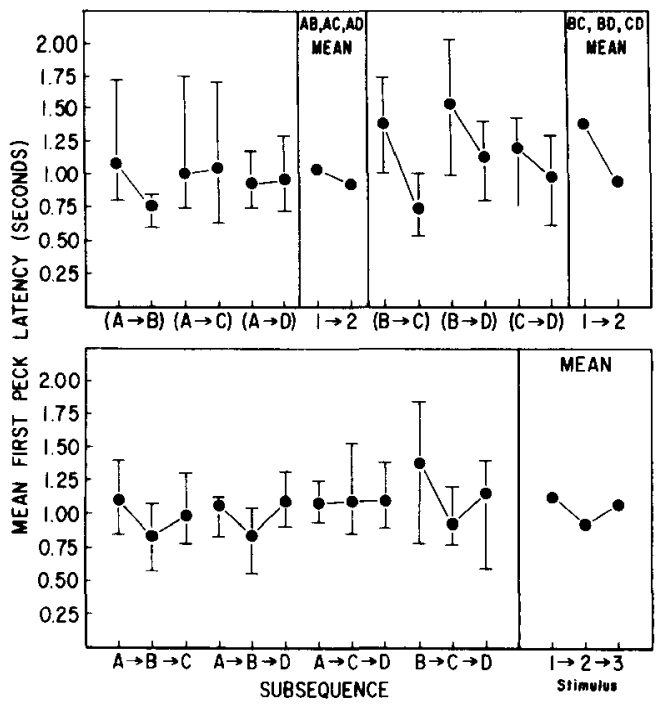

Figure 8. Average latencies of response to each color for each two- and three-color subsequence. Vertical lines indicate range of each distribution. Upper panel: two-color subsequences. Lower panel: three-color subsequences. 
spectively. The latencies to $A$ are very similar to those observed during $A \rightarrow B$ training (cf. Table 3). The upper portion of Figure 8 also shows that latencies to the second element of a subsequence beginning with $\mathrm{B}$ or $\mathrm{C}$ were shorter than latencies to the first element $[\mathrm{t}(6)=2.95, \mathrm{p}<.05]$.

The upper portion of Table 6 presents the average number of repetitive responses and the average time spent on repeats (dwell time), averaged for each subsequence across all subjects. On transitions between adjacent colors $(A \rightarrow B, B \rightarrow C, C \rightarrow D)$ and nonadjacent colors $(A \rightarrow C, A \rightarrow D$, and $B \rightarrow D)$, there were 1.8 and 2.5 repeats, respectively $[t(6)=2.59, p<.05]$. Dwell time on repeats averaged .86 and $1.15 \mathrm{sec}$ during transitions between adjacent and nonadjacent colors, respectively $[\mathrm{t}(6)=2.9, \mathrm{p}<.05]$.

\section{Three-Color Subsequence Test}

The proportion and range of trials completed correctly for each subsequence during the first test session is shown in the upper right-hand portion of Figure 7. All subjects completed correctly a greater number of trials containing each subsequence than predicted by random-choice responding [expected number $=13.6(80 \times .170)]$ with completely random choices $[\mathrm{t}(3)=3.13, \mathrm{p}<.05]$. The rank order of difficulty of the three-color subsequences (as measured by the average number of subsequences completed correctly by all subjects) was: $A \rightarrow B \rightarrow D, 65.94 \%$; $A \rightarrow$ $B \rightarrow C, 53.57 \% ; A \rightarrow C \rightarrow D, 41.73 \%$; and $B \rightarrow C \rightarrow D$, $39.13 \%$. The average proportion of trials correctly completed during the first five trials with each subsequence was as follows: $A \rightarrow B \rightarrow C, 57.14 ; A \rightarrow B \rightarrow D$, 68.57; $A \rightarrow C \rightarrow D, 42.86 ;$ and $B \rightarrow C \rightarrow D, 22.86$.

The lower portion of Figure 7 shows the average

Table 6

Average Number and Time (in Seconds) Spent on Repeats During Two- and Three-Color Subsequence Test

\begin{tabular}{|c|c|c|c|c|}
\hline \multicolumn{2}{|c|}{$\begin{array}{l}\text { Two-Color } \\
\text { Subsequence }\end{array}$} & \multicolumn{2}{|l|}{$\begin{array}{c}\text { Mean } \\
\text { Repeats }\end{array}$} & $\begin{array}{l}\text { ean Time on } \\
\text { Repeats }\end{array}$ \\
\hline \multirow{2}{*}{\multicolumn{2}{|c|}{$\begin{array}{l}A \rightarrow B \\
B \rightarrow C \\
C \rightarrow D\end{array}$}} & \multicolumn{3}{|c|}{ Adjacent Subsequence } \\
\hline & & $\begin{array}{r}.99 \\
1.85 \\
2.43\end{array}$ & \multicolumn{2}{|r|}{$\begin{array}{l}.32 \\
.52 \\
.86\end{array}$} \\
\hline & & \multicolumn{3}{|c|}{ Nonadjacent Subsequence } \\
\hline \multicolumn{2}{|c|}{$\begin{array}{l}\mathrm{A} \rightarrow \mathrm{C} \\
\mathrm{A} \rightarrow \mathrm{D} \\
\mathrm{B} \rightarrow \mathrm{D}\end{array}$} & \multicolumn{2}{|l|}{$\begin{array}{l}1.94 \\
2.01 \\
3.92 \\
\end{array}$} & $\begin{array}{r}.89 \\
.77 \\
1.33 \\
\end{array}$ \\
\hline \multirow{2}{*}{$\begin{array}{c}\text { Stim- } \\
\text { ulus }\end{array}$} & \multicolumn{4}{|c|}{ Three-Color Subsequence } \\
\hline & $\mathrm{A} \rightarrow \mathrm{B} \rightarrow \mathrm{C}$ & $\mathrm{A} \rightarrow \mathrm{B} \rightarrow \mathrm{D}$ & $A \rightarrow C \rightarrow D$ & $\mathrm{~B} \rightarrow \mathrm{C} \rightarrow \mathrm{D}$ \\
\hline & \multicolumn{4}{|c|}{ Repeats } \\
\hline \multirow[t]{2}{*}{2} & $\begin{array}{l}1.12 \\
2.25\end{array}$ & $\begin{array}{r}.79 \\
3.56\end{array}$ & $\begin{array}{l}1.59 \\
1.81\end{array}$ & $\begin{array}{l}2.81 \\
1.64\end{array}$ \\
\hline & \multicolumn{4}{|c|}{ Mean Time on Repeats } \\
\hline $\begin{array}{l}1 \\
2\end{array}$ & $\begin{array}{l}.374 \\
.570\end{array}$ & $\begin{array}{r}.212 \\
1.368\end{array}$ & $\begin{array}{l}.623 \\
.598\end{array}$ & $\begin{array}{l}.663 \\
.542\end{array}$ \\
\hline
\end{tabular}

proportion of responses made to the correct color at each position of the three-color subsequences. Again, the most difficult subsequence $(B \rightarrow C \rightarrow D)$ results from inaccuracy in responding to color $B$ as a first choice. As in the two-color subsequences test, poor performance on subsequences that required $B$ as a first response cannot be attributed to a failure to discriminate $B$ from the other colors. Accuracy to B as a second choice stimulus $(A \rightarrow B \rightarrow C, A \rightarrow B \rightarrow D)$ was much higher than it was to $B$ as a first-choice stimulus. As a second choice stimulus, the mean accuracy to $B$ across subjects was $86.76 \%$; as a first choice stimulus, it was $51.75 \%$ [t(6) $=5.02, \mathrm{p}<.005]$.

An increase in the accuracy of responding to the first color with increasing distance of the second color of the subsequence (as defined by the $A \rightarrow B \rightarrow$ $C \rightarrow D$ sequence) was also observed in the case of threecolor subsequences. On $A \rightarrow B \rightarrow C, A \rightarrow B \rightarrow D$, and $A \rightarrow C \rightarrow D$ subsequences, the average accuracy of responding to $A$ was $74.28 \%, 84.05 \%$, and $94.21 \%$, respectively $[A \rightarrow C \rightarrow D$ vs. the others combined: $t(6)=4.40, p<.005$ ]. Similarly, accuracy to $B$ increased from $78.90 \%$, in the case of $A \rightarrow B \rightarrow C$ subsequences, to $94.62 \%$, in the case of $A \rightarrow B \rightarrow D$ subsequences $[t(6)=3.78, p<.005]$.

All subjects showed a sharp decline in accuracy to color $C$ in $A \rightarrow C \rightarrow D$ subsequences. This is inconsistent with performance on $A \rightarrow C$ subsequences presented during the two-color test. $\mathrm{A} \rightarrow \mathrm{C}$ arrays occasioned the second highest accuracy of performance on two-color subsequences.

The lower portion of Figure 8 presents the means and the ranges of latencies of pecking each color for each subsequence. The latency to $A$ is quite constant: $1.09,1.08$, and $1.05 \mathrm{sec}$ on $\mathrm{A} \rightarrow \mathrm{B} \rightarrow \mathrm{C}, \mathrm{A} \rightarrow \mathrm{B} \rightarrow \mathrm{D}$, and $A \rightarrow C \rightarrow D$ sequences, respectively. In the one subsequence beginning with $A$ in which the second color was nonadjacent $(A \rightarrow C \rightarrow D)$, the latency of the response to the second color was longer than it was in subsequences in which the second color was adjacent $(A \rightarrow B \rightarrow C, A \rightarrow B \rightarrow D)[t(6)=3.0, p<.05]$. When $B$ was the first color to peck ( $B \rightarrow C \rightarrow D$ sequences), the latency of first pecks increased to $1.364 \mathrm{sec}$ [latency to $A$ vs. B as first responses, $t(3)=3.34, p<.001$ ].

The lower portion of Table 6 shows the average number of repetitive responses and the average dwell time at each sequence position for all subsequences. On transitions between adjacent and nonadjacent colors, there were 1.76 and 2.57 repeats, respectively $[t(6)=2.53, p<.05]$. Dwell time on repeats was .49 and $.99 \mathrm{sec}$ during transitions between adjacent and nonadjacent colors, respectively $[t(6)=2.94, p<.05]$.

\section{DISCUSSION}

The results of Experiment 1 replicate and extend our earlier demonstration of serial learning in pigeons. Even though each of the three subjects in the Straub 
et al. study learned to respond to arrays of A, B, C, and $D$ at $60 \%$ accuracy, it was not possible to obtain orderly acquisition functions because of the variety of training procedures (backward chaining, a correction procedure, and feedback for correct responses) that were used before the paradigm that was followed throughout this study was put into effect. Figure 1 shows that forward training, consisting of successive exposure to $\mathrm{AB}, \mathrm{ABC}$, and $\mathrm{ABCD}$ arrays, results in orderly acquisition of $A \rightarrow B, A \rightarrow B \rightarrow C$, and $A \rightarrow B \rightarrow$ $C \rightarrow D$ sequences.

Experiment 1 also shows unequivocally that, by the end of training on $A B C D$ arrays, it was the sequence $A \rightarrow B \rightarrow C \rightarrow D$ that was learned rather than particular configurations of training arrays. Indeed, complete generalization was observed during the first five test trials of the tests given after both the $50 \%$ and the $70 \%$ accuracy criteria were met.

The subjects of the Straub et al. experiment performed at better than chance levels of accuracy on novel arrays. They nevertheless performed at a lower level of accuracy on novel arrays than on training arrays. This indicates that, to some extent, their performance was based upon familiarity with the training arrays. The most compelling explanation of an absence of a generalization decrement on novel arrays in Experiment 1 is the larger number of training arrays used in this study (80 vs. 16).

The results of Experiment 2 show that, in acquiring the sequence $A \rightarrow B \rightarrow C \rightarrow D$, our subjects learned about relationships between nonadjacent as well as adjacent elements. Had they not done so, they could not have responded correctly to subsequences such as BD in which the first element (A) and a subsequent adjacent element $(C)$ had been omitted. These and other aspects of our results, which are not readily explainable by S-R modes of chaining theory, suggest that, in learning to perform a simultaneous chain such as $A \rightarrow B \rightarrow C \rightarrow D$, pigeons are able to make use of an internal representation of the elements $\mathrm{A}, \mathrm{B}, \mathrm{C}$, and $D$.

Before considering how a pigeon might represent a sequence of elements, it is important to establish clearly the shortcomings of traditional S-R theory in accounting for the serial learning demonstrated in the current study. We will evaluate our results with respect to two aspects of chaining theory: the mechanism it postulates for integrating a series of seemingly separate responses and the predictions it makes regarding the relative response strengths of different members of chain.

(a) The integration of successive responses: Each stimulus elicits (or occasions) a response and (except the first stimulus) also functions as a secondary reinforcer for the response that produced it. At each step of the chain, the occurrence of a particular response is assumed to be determined uniquely by the presence of a particular stimulus complex. This view suggests that the sequence of responses that defines a chain is an automatic consequence of environmental contingencies which insure that particular stimuli occur in a particular order.

Consider, for example, the response to stimulus $B$ in the successive chain $A \rightarrow B \rightarrow C \rightarrow D$. It is assumed that the occurrence of response $B$ depends solely on the presence of stimulus B and the appropriate manipulandum. Note that proprioceptive feedback from response $\mathbf{A}$ is irrelevant. A simultaneous chain, in which the position of each element varies from trial to trial, provides no basis for a unique response $A$.

The absence of step-by-step feedback from a simultaneous chaining paradigm poses a serious problem for chaining theory, since the external environment remains unchanged as the chain is executed. It might be argued, however, that our subjects learned to respond to a sequence of self-generated discriminative stimuli. Consider a pigeon's response to a single color which can appear on any one of six response keys. It is reasonable to expect that a pigeon would learn to peck that color wherever it appeared. Accordingly, the discriminative stimulus would be defined as a color, say $\mathbf{A}$, irrespective of its position. If $A$ is now embedded in arrays of other stimuli (B, $C$, and D), all that the pigeon needs to learn at the onset of the trial is to peck A (as previously) and to ignore the other elements. Now suppose that the "act of pecking A" (or "the sight of A while pulling away from the key") comes to serve as a discriminative stimulus for pecking B. Likewise, the "sight of B" during the recoil of a peck to $B$ might come to serve as a discriminative stimulus for pecking $C$, and so on. Thus, the pigeon creates a successive chain from simultaneously presented elements and, in so doing, it insures the kind of step-by-step feedback that a successive chaining paradigm typically provides.

Logically, such an interpretation is defensible; practically, it is highly implausible. We are aware of nothing in the conditioning literature that suggests that a pigeon might learn a set of rules of the form "when in the presence of one element, peck some particular other element" without explicit differential reinforcement for doing so at each step.

The results of Experiment 2 cannot be explained by chaining theory, even on logical grounds. Consider accurate performance on a subset such as BD. One cannot argue that the subject looks for $A$ at the start of the trial, and, having pecked $B$, that the sight of $B$ is a discriminative stimulus for pecking $D$. Aside from accurate performance on subsets containing nonadjacent elements, or which do not start at $\mathbf{A}$, our latency data suggest that, when a normally available element is absent, the bird uses some sort of representation of the sequence as an aid in selecting the next element. The latency of the first response to 
two-element arrays beginning with $\mathrm{B}$ was longer than those beginning with $\mathrm{A}$ (cf. Figure 8, upper portion). Likewise, the bottom portion of Figure 8 shows that the latency of the first response to the three-element array beginning with $\mathrm{B}$ (BCD) was longer than the latency of the first response to three-element arrays beginning with $A(A B C, A B D$, and $A C D$ ).

(b) Response strength of different members of simultaneous chain: Both Hull's (1943) and Skinner's (1938) models of chaining assume that the last response of the chain (i.e., the response that is followed by primary reinforcement) is strongest and that the first response is weakest. This state of affairs follows directly from a delay of reinforcement gradient. Neither the results of the present experiment nor those of our initial study confirm such predictions. The relevant data include the manner in which the chain was trained, the relative frequency of oneand two-skip forward errors, their latencies, and the relative frequency of repeat pecks to each element of the sequence.

Straub et al. (1979) attempted to train the simultaneous chain $A \rightarrow B \rightarrow C \rightarrow D$ by first training a peck to $D$, then requiring that pecking occur in the sequence $C \rightarrow D$, then in the sequence $B \rightarrow C \rightarrow D$, and, finally, in the sequence $A \rightarrow B \rightarrow C \rightarrow D$. This procedure proved useless during the second phase of training $(C \rightarrow D)$. Having been trained initially to peck $D$, our subjects persisted in pecking $D$ at the onset of $C D$ arrays. That, of course, was a one-skip forward error which resulted in the offset of the trial without reinforcement. Nonreinforced pecking to $D$ persisted until it was extinguished. In the case of a successive chaining paradigm, it is easy to see how working backward from reinforcement would prove more successful. Once a response has been shaped to $D$, $D$ is withheld until a response to $C$ occurs. Since $C$ and $D$ are never available simultaneously in a successive chain, the problem of a prepotent response to $D$ does not exist.

A chaining model predicts progressively shorter latencies and more repeat pecks as the subject moves through the sequence. It also predicts more twoskip than one-skip errors and more three-skip than two-skip errors. These predictions follow from the differential amount of response strength that accrues from different delays of reinforcement for responding to $A, B, C$, and $D$, respectively.

A pattern of decreasing latencies to successive elements of the sequence could not be discerned at the end of either three- or four-element training. At the end of three-element training, the average latencies of correct responses to $\mathrm{A}, \mathrm{B}$, and $\mathrm{C}$ were $1.093, .867$, and $1.167 \mathrm{sec}$, respectively. At the end of fourelement training, the average latencies of correct responses were $.990, .743, .798$, and $.941 \mathrm{sec}$, respectively.
The number of repeat pecks increased and then decreased as the sequence was performed. At the end of $A \rightarrow B \rightarrow C$ training, the relative frequency of repeat pecks increased as the subject moved from $A$ to $B$ : at $A, 42.16 \%$ of all pecks were repeats; at $B, 69.41 \%$ were. During $A \rightarrow B \rightarrow C \rightarrow D$ training, however, the relative frequency of repeat pecks to $\mathrm{A}, \mathrm{B}$, and $\mathrm{C}$ were $44.61 \%, 66.87 \%$, and $29.52 \%$, respectively.

The relative frequency of forward errors decreased as the distance between the element last responded to and the element that defined the forward error increased. At the end of $A \rightarrow B \rightarrow C \rightarrow D$ training, the relative frequencies of one-, two-, and three-skip errors at $\mathrm{A}$ were $11.12 \%, 3.36 \%$, and $9 \%$, respectively; the relative frequency of one- and two-skip errors at $B$ were $4.40 \%$ and $1.36 \%$, respectively. Overall, there was no evidence that any measure of response strength increased as the subject moved closer to reinforcement.

We recognize that chaining theory can account for certain features of these data by appealing to interactions between gradients of excitation around each response or to unrecorded behavior. Hull (1952), for example, derived U-shaped functions relating latency to position in the sequence (of a successive chain) by postulating interactions between hypothetical gradients of excitation at each position. It is not clear how this approach predicts other aspects of our data, for example the monotonic decrease in the size of forward errors at each step of the sequence. In any event, the paucity of empirical evidence of different types of interactions between gradients of excitation and inhibition of varying magnitudes, at different points in a serial task, makes one question the explanatory value of such ad hoc hypotheses.

A more plausible basis for explaining certain features of our data, such as the relative frequencies of forward errors, derives from informal observations of "air pecks" that occurred occasionally when our subjects made forward errors. An air peck is a peck directed at the key that fails to operate the key. Suppose that a two-skip forward error (such as $A \rightarrow D$ ) results from a peck to $A$, air pecks to $B$ and $C$, and a peck to $D$. If we assume that air pecks occur with a certain probability and that successive air pecks are independent events, it follows that the probability of two successive air pecks should be lower than the probability of one air peck, an outcome that is consistent with our data. It should be recognized, however, that extensive observations of our subjects revealed many one- and two-skip errors that could not be attributed to air pecks. That is, the subjects appeared to proceed directly from element $n$ to element $\mathbf{n}+2$ without an intervening air peck.

(c) Possible strategies for executing a simultaneous chain: Our present data are too meager to substantiate the few hypotheses that can be suggested as to how our 
subjects represented the sequences they performed. Nevertheless, we feel that the theoretical vacuum left by the failure of chaining theory is justification enough for speculating about the strategies that our subjects may have followed in performing a simultaneous chain.

In the case of two-element subsets, it might be argued that all the bird needs to do is peck the first element correctly, and then, by default, peck the second element, whatever that element might be. This argument begs the question of how the bird manages to peck the first element correctly when it is not $A$. It also does not explain why the latency of the response to the second element of BD arrays was shorter than the latency of the response to the second element of $B C$ arrays. One possibility is that in learning the sequence $\mathrm{A} \rightarrow \mathrm{B} \rightarrow \mathrm{C} \rightarrow \mathrm{D}$ the bird learns two rules that enable it to respond correctly to nonadjacent elements: (1) start at A, (2) respond to D last. Rule 2 would account for performance on BD arrays. It would not, however, do for BC arrays. Since performance was least accurate on $\mathrm{BC}$ arrays, we cannot definitely rule out the possibility that our subjects were using rules 1 and 2 in performing two-element subsets.

The default strategies described by rules 1 and 2 can be generalized to three-element subsets. On arrays such as $A C D$, the application of rule 1 leaves the bird with a choice of $C$ and $D$. If the bird then applies rule 2, C is the only element left to peck. Having done so, another application of rule 2 would result in the successful completion of the sequence. The performance of our subjects on arrays such as ACD provides evidence that they used different strategies in responding to adjacent and nonadjacent elements. The latency of the response to the second element of $A C D$ arrays was longer than the latency of the second response to $A B C$ and $A B D$ arrays.

While applicable to subsequences derived from the four-element sequence $A \rightarrow B \rightarrow C \rightarrow D$, rules 1 and 2 need to be supplemented to account for accurate performance on subsequences derived from longer sequences. Consider, for example, the eight-element sequence $A \rightarrow B \rightarrow C \rightarrow D \rightarrow E \rightarrow F \rightarrow G \rightarrow H$. Accurate performance on two-element subsequences such as $C F$ or DG could not be explained by any application of rules 1 and 2 . Similar problems would be posed by accurate performance on longer subsequences such as $B \rightarrow E \rightarrow G$ or $B \rightarrow D \rightarrow F$. In the case of these and other subsequences, one might postulate additional rules such as: (3) B and C are "early" elements, (4) D and $E$ are "middle" elements, (5) $F$ and $G$ are "late" elements, and (6) respond to $\mathrm{H}$ last. That these or related rules are used by a pigeon in performing longer simultaneous chains will, of course, have to be demonstrated by the features of performance on subsets derived from sequences longer than the $A \rightarrow B \rightarrow C \rightarrow D$ sequence used in the present experiment. At present, however, rules such as 1-6 appear to provide plau- sible and parsimonious alternatives to a chaining model of sequential performance.

A sizable literature on human serial learning has questioned the adequacy of a chaining model in accounting for the acquisition of verbal sequences and has explored various alternative models (e.g., Giurintano, 1973; Jensen \& Rohwer, 1965; Primoff, 1938; Shuell \& Keppel, 1967; Stark, 1968). It has been suggested that human subjects who learn a serial task (cf. Ladd \& Woodworth, 1911; Young, 1961) learn to associate particular elements with particular positions of the sequence, that is, A with the first position, B with the second, and so on. An ability to order the relative positions of a series of elements would, of course, account for our subjects' ability to respond correctly to subsets that omit various elements. Hulse's demonstrations of the rat's ability to learn the overall organization of a series of food pellets of decreasing magnitude (cf. Hulse, 1978; Hulse \& Dorsky, 1977) suggests some ability on the part of nonprimates to order the elements of a sequence. It remains to be seen, however, just what relationships of order a pigeon can learn about a sequence of elements. A pigeon might learn that A precedes $B, C$, and $D$, that $B$ precedes $C$ and $D$, and that $C$ precedes $D$, and still be unable to associate a particular element with a particular position of an ABCD sequence.

Before applying models of human serial learning to the execution of simultaneous chains by pigeons, one should keep in mind that implicit in all models of human serial learning is the ability of the subjects to interpret symbolically each element of the sequences they are required to learn. Until evidence to the contrary is available, it may prove foolhardy to base models of a pigeon's representation of a sequence on a human's ability to learn sequences. At the same time, it is of interest to note that a nonverbal creature such as a pigeon has the competence to respond in the appropriate sequence to subsets of a previously learned list of elements, and that such performance implies control by internal processes that cannot be explained as S-R chains. An understanding of such processes may not only prove valuable in explaining sequential behavior of lower organisms but may also provide an important benchmark for evaluating human serial performance.

\section{REFERENCES}

Broadbent, D. E. Behavior. New York: Basic Books, 1961. Сномsкy, N. Syntactic structures. The Hague: Mouton, 1957. Chomsky, N. A review of Skinner's "Verbal Behavior." Language, 1959, 35, 26-58.

Fodoh, J. A., Bever, T. G., \& GarnetT, M. F. The psychology of language: An introduction to psycholinguistics and generative grammar. New York: McGraw-Hill, 1974.

Giurintano, S. L. Serial learning process: Test of chaining, position, and dual-process hypotheses. Journal of Experimental Psychology, 1973, 97, 154-157. 
Guthrie, E. R. The psychology of learning. New York: Harper \& Row, 1952.

HuLl, C. L. Principles of behavior. New York: Appleton-CenturyCrofts, 1943.

Huzse, S. H. Cognitive structure and serial pattern learning by animals. In S. H. Hulse, H. Fowler, \& W. K. Honig (Eds.), Cognitive processes in animal behavior. Hillsdale, N.J: Erlbaum, 1978.

Hulse, S. H., \& Donsky, N. P. Structural complexity as a determinant of serial pattern learning. Learning and Motivation, 1977, 8, 488-506.

Jensen, A. R., \& Rohwer, W. D. What is learned in serial learning? Journal of Verbal Learning and Verbal Behavior, $1965,4,62-72$.

LADD, G. T., \& WoODWorth, R. S. Elements of physiological psychology. New York: Scribner's, 1911.

LABHLEY, K. S. The problem of serial order in behavior. In L. A. Feffries (Ed.), Cerebral mechanisms in behavior. New York: Wiley, 1951.

LAshley, K. S., \& BALL, J. Spinal conduction and kinesthetic sensitivity in the maze habit. Journal of Comparative and Physiological Psychology, 1929, 9, 71-10s.

Miller, G. A., Galanten, E., \& Pribram, K. H. Plans and the structure of behavior. New York: Holt, Rinehart \& Winston, 1960.

Outon, D. S. Characteristics of spatial memory. In S. H. Hulse, H. Fowler, W. K. Honig (Eds.), Cognitive processes in animal behavior. Hillsdale, N.J: Erlbaum, 1978.

Olton, D. S. Mazes, maps and memory. American Psychologist, $1979,34,588-596$.

Olton, D. S., \& SAmuelson, R. J. Remembrance of places past: Spatial memory in rats. Journal of Experimental Psychology: Animal Behavior Processes, 1976, 2, 97-116.

Primorf, E. Backward and forward association as an organizing act in serial and in paired associate learning. Journal of Psychology, 1938, 5, 375-395.

RiLey, D. A. Comments on Simon's paper and some observations on information processing in animals. In $\mathbf{M}$. R. Rosenzweig \& E. L. Bennett (Eds.), Neural mechanisms of learning and memory. Cambridge, Mass: M.I.T. Press, 1976.

Shepard, R. N. Form, formation and transformation of internal representations. In R. L. Solso (Ed.), Information processing and cognition. Hillsdale, N.J: Erlbaum, 1975.

Shuell, T. J., \& Keppel, G. A further test of the chaining hypothesis of serial learning. Journal of Verbal Learning and Verbal Behavior, 1967, 6, 439-445.

Sxinner, B. F. The behavior of organisms. New York: AppletonCentury-Crofts, 1938.

STARK, K. Transfer from serial to paired-associate learning: A reappraisal. Journal of Verbal Learning and Verbal Behavior, $1968,7,20-30$.

Straub, R. O., Seidenberg, M. S., Bever, T. G., \& Terrace, H. S. Serial learning in the pigeon. Journal of the Experimental Analysis of Behavior, 1979, 32, 137-148.

Terrace, H. S., Straub, R. O., Bever, T. G., \& Seidenberg, M. S. Representation of a sequence by a pigeon. Bulletin of the Psychonomic Society, 1977, 10, 269.

Youna, R. K. A comparison of two methods of learning serial associations. American Journal of Psychology, 1959, 72, 554-559.

Youna, R. K. The stimulus in serial verbal learning. American Journal of Psychology, 1961, 74, 517-528.

\section{NOTE}

1. Pretraining on adjacent elements for the subjects of Group 2 was discontinued after 16 sessions. At that point in training, only one bird (No. 298) consistently completed at least $50 \%$ of the trials (chance performance) with any of the arrays $(C \rightarrow D$ and $A \rightarrow B$ ). Differences in the proportion of trials correctly completed by subjects of Group 2 with each pretraining sequence are insignificant $[F(2,6)=2.20, p>.05]$. The average percentages of correctly completed $A \rightarrow B, B \rightarrow C$, and $C \rightarrow D$ trials across subjects for the last five pretraining sessions were: $48.32 \%, 38.06 \%$, and $58.94 \%$, respectively. Pilot data from a group of four birds receiving more extended training on these sequences corroborates the ranking of array difficulty. At every stage in training, the order of arrays, ranked from most to least difficult, was $B \rightarrow C, A \rightarrow B, C \rightarrow D$. Greater-than-chance performance with all three sequences was observed only after 50-60 sessions.

(Manuscript received December 5, 1980; revision accepted for publication July 22,1981 .) 